Peer-reviewed research

\title{
Has COVID-19 Changed Exchange Rate Resistance to Shocks?
}

\author{
Paresh Kumar Narayan ${ }^{1}$ a \\ 1 Deakin university, Australia \\ Keywords: exchange rate, shocks, yen-us dollar, covid-19 \\ https://doi.org/10.46557/001c.17389
}

\section{Asian Economics Letters}

Vol. 1, Issue 1, 2020

In this note, we propose the hypothesis that COVID-19 has influenced the Yen-US dollar exchange rate's resistance to shocks. We propose a time varying unit root model and unravel that prior to the pandemic, the Yen was non-stationary while during the pandemic it became mostly stationary, suggesting that shocks to the Yen are having a transitory effect. We, as a result, conclude that COVID-19 has changed the resistance of the Yen to shocks.

\section{Introduction}

One of the most affected asset prices due to COVID-19 is the exchange rate; see Iyke (2020). In this note, we test whether shocks to exchange rate (the Japanese Yen-US dollar) have a permanent or transitory effect. Our hypothesis, motivated by recent literature on COVID-19's effect on the financial system, is that shocks to exchange rates are likely to have a transitory effect. This hypothesis test is motivated by the theory of efficient market hypothesis (EMH). The idea is that if markets are inefficient traders can extract profits (Iyke, 2019). Inefficiency or otherwise can be judged through a test of unit roots. The second theory that motivates our empirical exercise is that of purchasing power parity (PPP). A unit root test that confirms stationary exchange rate implies existence of PPP. The relevance of finding PPP to economic theories have been well covered by the literature and we avoid repeating this discussion; interested readers are referred to Narayan (2006).

The COVID-19 pandemic represents the largest global shock (Phan \& Narayan, 2020). In light of how COVID-19 has influenced and continues to influence the global financial system, it is natural to ask how it has affected exchange rates. Our question is positioned within the unit root literature motivated by the EMH and the PPP theory. Our goal is to capture the effect of COVID-19 on the effect of shocks on exchange rates. While for application purpose any exchange rate can be used, we prefer using the Yen-US dollar exchange rate because the Yen is amongst the very few currencies that have appreciated vis-à-vis the US dollar over the COVID-19 period; other reasons for studying the Japanese market have been discussed in a recent paper by Narayan et al. (2020) and we refer interested readers to that paper. To capture any possible effects of COVID-19, we fit hourly Yen -US dollar exchange rate data, spanning the period 7/01/2019 to 9/04/2020 to the Narayan and Popp (2010) two endogenous structural break unit root model. However, to test our hypothesis, given that COVID-19 is a recent phenomenon, we need a time-varying model to capture its effects. We, therefore, extend the NP test to a time-varying one.

Our main result is that from 3/30/2020 04:00 to 9/04/ $202017: 00$, of the 1,951 expanding windows, the unit root null is rejected in $45.3 \%$ of the sample. By comparison, prior to $3 / 30 / 2020$, there are 2,265 expanding windows and the unit root null is only rejected in $1.9 \%$ of the sampled windows. This tells that shocks to the Yen exchange rate have become short-term or transitory in the COVID-19 phase. Our contribution to the literature is precisely in understanding the effects of the COVID-19 pandemic on exchange rate behavior. Except for Iyke (2020), who studies exchange rate predictability using COVID-19 cases of the virus and shows evidence of predictability, none of the studies have explored any other aspect of exchange rate response to COVID-19. While we complement Iyke (2020), our contribution is distinct in the sense that we evaluate the resistance of the exchange rate to the pandemic. Another difference between our paper and Iyke (2020) is that their modelling is based on predictability while ours is about unit roots.

\section{Data and results}

\section{A. Data}

In this note, we use hourly Yen-US dollar exchange rate covering the sample 7/01/2019 01:00 to 9/04/2020 17:00, culminating into a total of 5,270 observations. The exchange rate is in terms of Yen per one US dollar. A plot of the data is provided in Figure I. Several observations make way. First, the Yen exchange rate has two major phases-a pre-COVID-19 and a COVID-19 phase as marked on the graph. Six months before the pandemic, the Yen had depreciated, from 105.12 Yen per US dollar to 109.78 Yen per US dollar. In this pre-COVID-19 period, the average exchange rate was 108 Yen per US dollar. The Yen-US dollar standard deviation was 1.098 .

Second, by comparison, during COVID-19, on average, the exchange rate was at 107.22, suggesting that on average the Yen appreciated in the COVID-19 period compared to the pre-COVID-19 period. Third, we see that the Yen was

\footnotetext{
a Corresponding Author Professor Paresh Kumar Narayan Centre for Financial Econometrics Faculty of Business and Law Deakin University 221 Burwood Highway Burwood, Victoria 3125 Australia Telephone: +61 392446180 Fax: +61 392446034 Email: paresh.narayan@deakin.edu.au
} 


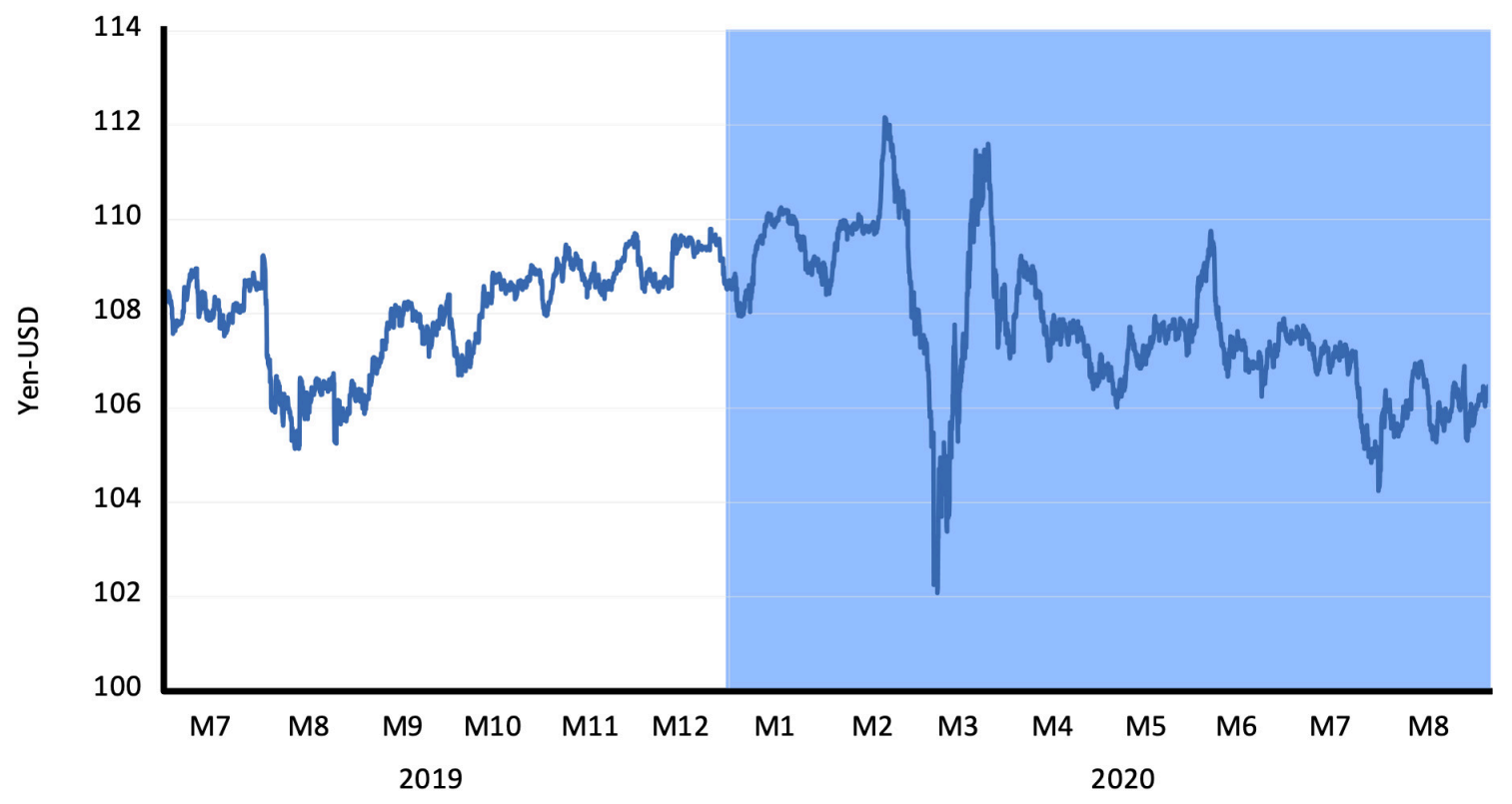

Year

Figure I: Japanese Yen-US dollar exchange rate

This figure plots hourly Yen-US dollar exchange rate where an increase represents a depreciation of the Yen. The hourly data cover the sample 7/01/2019 01:00 to 9/04/2020 17:00 for a total of 5,270 observations.

more volatile during the COVID-19 period, with a standard deviation of 1.609. Fourth, the appreciation of the Yen during the pandemic has been stronger, from 112.15 Yen per US dollar to 102.06 . The question is: did this higher volatility and greater appreciation of the Yen in the COVID-19 period have implications for its resistance to other shocks during the pandemic? A time-varying unit root model can answer this question and we turn to it next.

\section{B. Model and results}

We consider the NP unit root model that takes breaks only in the level and not in the trend. The motivation is simple: an inspection of Figure 1 tells of no trend. A timetrend ordinary least squares regression model produces a time trend slope coefficient of $-0.0002(t$-statistic $=-0.756)$. We, therefore, settle on the following model:

$$
\begin{aligned}
Y E N_{t}= & \alpha+\lambda Y E N_{t-1}+\beta t+\kappa_{1} B R_{B, 1}^{\prime} \\
& +\kappa_{2} B R_{B, 2}^{\prime}+\delta_{1} D U_{1, t-1}^{\prime} \\
& +\delta_{2} D U_{2, t-1}^{\prime}+\sum_{j=1}^{k} \beta_{j} \Delta Y E N_{t-j}+e_{t}
\end{aligned}
$$

In this model, YEN is the Yen-US dollar exchange rate where an increase denotes depreciation of the Yen vis-à-vis the US dollar; the optimal lag length, $k$, is chosen using the Schwarz Information criterion, where $\beta_{j}$ controls for any serial correlation; $t$ represent the trend; $e_{t}$ represents the model's residuals; $D T_{B, 1}$ and $D T_{B, 2}$ denote, respectively, the first and second break dates; and $D U_{1}$ and $D U_{2}$ are the level break dummy variables. These dummy variables have the following representation:

$$
D U_{i, t}^{\prime}=1\left(t>T_{B, i}^{\prime}\right)
$$

The unit root null hypothesis of $\lambda=1$ is tested against the alternative hypothesis that $\lambda<1$. The critical values (CV) of the test statistic are given in NP (2010).

Two additional choices need to be made. The first issue relates to the trimming factor to account for tails of the data. We use a $15 \%$ trimming factor, which for a large sample like ours is sufficient to account for outliers. Second, and most relevant to our hypothesis test, is the choice of the initial window to generate time-varying $\lambda$. There is no theory that dictates the size of the window. As a statistical rule, the window should be of sufficient size to generate parsimonious estimates of the null hypothesis of a unit root. With 5,270 observations, if we set the initial window to $20 \%$, this givens as an initial window sample size of 1,054 observations such that $T=1054$ and subsequent windows are set as $T+1$ and the model is estimated until $T=5,269+1$. We leave robustness tests on window size for future studies as our note is a demonstration of the technique.

The results are plotted in Figure II. In summary, we report both the slope coefficient of $\lambda$ representing the persistency of the Yen exchange rate and the associated $t$-statistic testing the null hypothesis of a unit root. If the slope coefficient falls it represents lack of persistency meaning that any shock to the Yen-US dollar will have a transitory effect and vice versa, conditional on rejection of the null hypothesis of a unit root. To test the unit root null of the NP two endogenous break unit root model, we utilize the $10 \% \mathrm{CV}$, which is -3.772. The CV is plotted as a dashed line to indicate statistical significance.

The model is estimated for the time period $7 / 01 / 2019$ 01:00 to $9 / 04 / 202017: 00$. This sample gives a total of 5,270 hourly observations. The model is estimated by setting the initial window to $20 \%$ of the sample. We then obtain a timevarying measure of persistency starting from 9/25/2019 01:00. The shaded region reflects the phase when COVID-19 was in the early stage and not much was known about its potential effects. We see during this time period the unit root null hypothesis is not rejected. This suggests that 


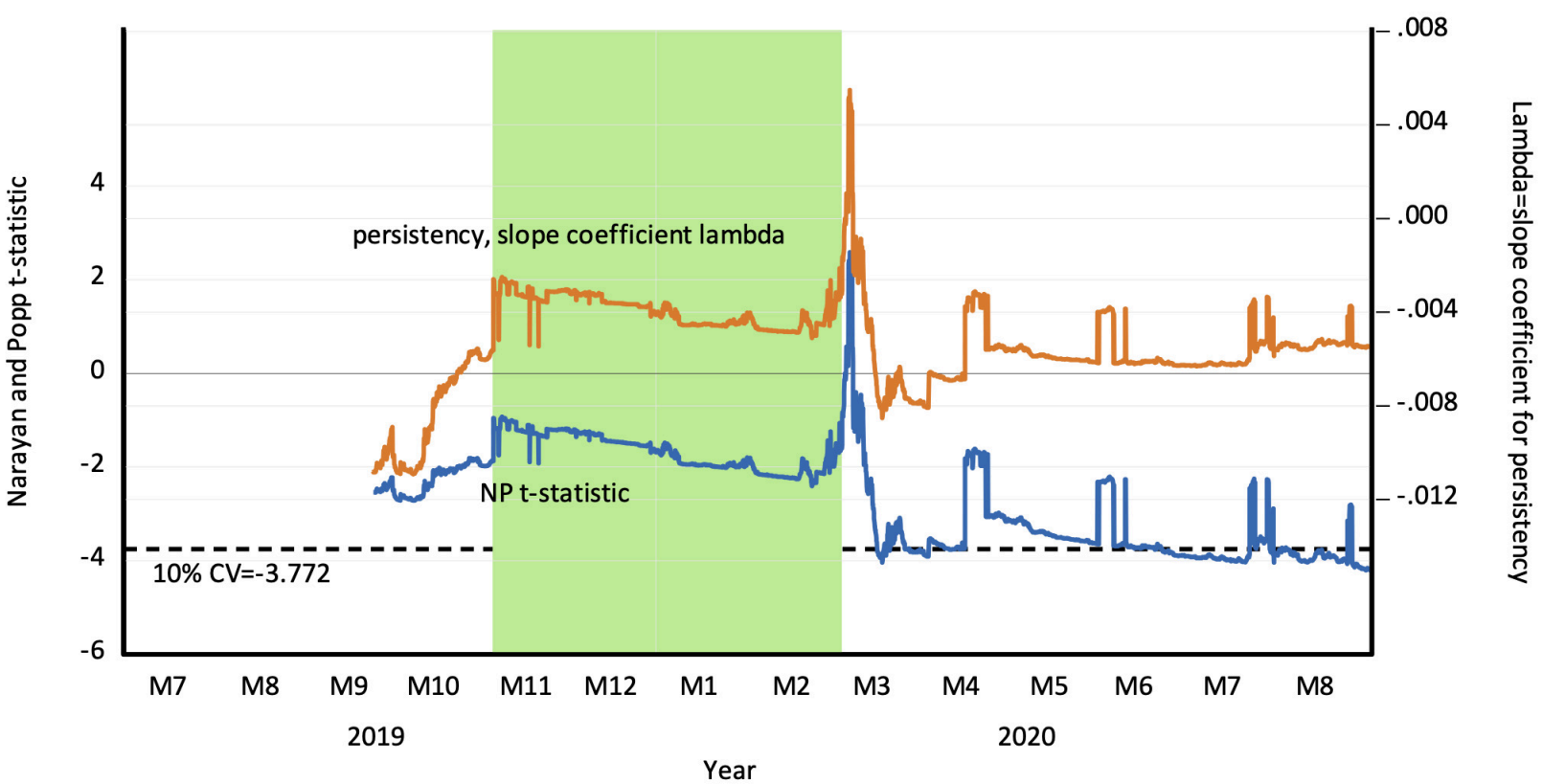

Figure II: Time-varying shock persistence of Yen-US dollar exchange rate

This figure plots the time-varying lambda-a measure of shock persistency-from the Narayan and Popp (NP, 2010) two endogenous break unit root model and the $10 \%$ critical value (CV) of the NP test of -3.772 . The CV is plotted as a dashed line to indicate statistical significance. The model is estimated for the time period 7/01/2019 01:00 to 9/04/2020 17:00. This sample gives 5,270 hourly observations. Two pre-requisites of the NP model are implemented: (a) a trimming region of $15 \%$ and (b) an initial estimation window made up of $20 \%$ of sample size. These conditions when implemented give us a starting sample date of persistency estimate of 9/25/2019 01:00. The shaded region reflects the phase when COVID-19 was in the early stage and not much was known about its potential effects.

shocks to Yen were having a non-transitory effect. However, after March, 2020; in other words, from March 30, 2020 to September 04, 2020, we observe that (a) the slope coefficients have become smaller and (b) most importantly, the unit root null hypothesis is rejected over most expanding windows in this COVID-19 phase.

More precisely, we see that in the 3/30/2020 04:00 period, there are 1,951 expanding windows. Out of this, in 883 windows the unit root null is rejected. This represents $45.3 \%$ of the sample. By comparison, in the pre-3/30/2020 period, there are 2,265 expanding windows and the unit root null is only rejected in 43 windows, representing only $1.9 \%$ of the sample. This tells that shocks to the Yen exchange rate became short-term or transitory in the COVID-19 phase. This is an interesting finding. Our explanation is as follows. COVID-19 itself is the largest global shock in history. It follows that any other shock should not matter, and this is what we observe from Figure II. In other words, over the COVID-19 phase (which is still in progress), the effect of any other shock (by way of news and/or market fundamentals) is modest and in the phase of COVID-19 is unlikely to persist.

\section{Concluding remarks}

In this note, we show how the time-varying Narayan and Popp (2010) two endogenous structural break unit root model can be utilized to understand the evolution of exchange rates from the point of view of shock persistency. Using the hourly Yen-US dollar exchange rate, we show that prior to the COVID-19 pandemic, the exchange rate was non-stationary; however, the Yen became highly stationary in the COVID-19 sample, suggesting that shocks to the Yen are having a transitory effect. We argue that this type of Yen behavior is because COVID-19 itself is the largest global shock and since COVID-19 is still unfolding other conventional shocks emanating from market fundamentals and/or news are trivial and unlikely to persist. Future studies can apply the time-varying model we present to study the effect of shocks on other financial assets.

\section{Acknowledgement}

Helpful comments and suggestions from an anonymous reviewer of this journal helped improve the paper. The usual disclaimer applies. The research is this paper is supported by funding obtained from the Asia-Pacific Applied Economics Association, Melbourne, Australia.

Submitted: September 22, 2020 AEDT, Accepted: September 28, 2020 AEDT

This is an open-access article distributed under the terms of the Creative Commons Attribution 4.0 International License (CCBY-SA-4.0). View this license's legal deed at https://creativecommons.org/licenses/by-sa/4.0 and legal code at https://creativecommons.org/licenses/by-sa/4.0/legalcode for more information. 


\section{REFERENCES}

Iyke, B. N. (2019). A test of the efficiency of the foreign exchange market in Indonesia. Buletin Ekonomi Moneter Dan Perbankan, 21, 439-464. http s://doi.org/10.21098/bemp.v0i0.976

Iyke, B. N. (2020). The disease outbreak channel of exchange rate return predictability: Evidence from COVID-19. Emerging Markets Finance and Trade, 56(10), 2277-2297. https://doi.org/10.1080/1540496 x.2020.1784718

Narayan, P. K. (2006). Are bilateral real exchange rates stationary? Evidence from Lagrange multiplier unit root tests for India. Applied Economics, 38(1), 63-70. https://doi.org/10.1080/00036840500365983
Narayan, P. K., Devpura, N., \& Hua, W. (2020). Japanese currency and stock market-what happened during the COVID-19 pandemic? Economic Analysis and Policy. https://doi.org/10.1016/j.eap.2020.09.014

Narayan, P. K., \& Popp, S. (2010). A new unit root test with two structural breaks in level and slope at unknown time. Journal of Applied Statistics, 37, 1425-1438. https://doi.org/10.1080/02664760903039 $\underline{883}$

Phan, D. H. B., \& Narayan, P. K. (2020). Country responses and the reaction of the stock market to COVID-19-a Preliminary Exposition. Emerging Markets Finance and Trade, 56(10), 2138-2150. http s://doi.org/10.1080/1540496x.2020.1784719 